\title{
Tangential Laser Turning of Fused Silica Using Ultra-short Laser Pulses
}

\author{
Julian Zettl ${ }^{*}$, Stefan Rung ${ }^{1}$, Cemal Esen ${ }^{2}$ and Ralf Hellmann ${ }^{1}$ \\ ${ }^{1}$ University of Applied Sciences Aschaffenburg, Applied Laser and Photonics Group, Würzburger \\ Strasse 45, 63743 Aschaffenburg \\ ${ }^{2}$ Ruhr University Bochum, Applied Laser Technologies, Universitätsstr. 150, 44801 Bochum \\ *Corresponding author's e-mail: Julian.Zettl@th-ab.de
}

\begin{abstract}
Laser turning with ultra-short laser pulses provides the possibility of manufacturing rotationally symmetric geometries, even of hard to machine materials as fused silica, down to a micrometer scale. In this laser micro machining approach, the laser beam is oriented perpendicularly to the rotational axis and tangentially to the material, whereas the target geometry is realized by moving the constantly rotating specimen according to the specified geometry under the focused laser spot. Due to the friction-less laser ablation it is possible to realize a turning process in fused silica without the use of a contacting tool and therefore without causing mechanical tensions in the work piece. The processing head focuses the laser to a calculated spot size of $18 \mu \mathrm{m}$ in diameter and the applied processing gas pressure assists in removing the ablated material. In this study, femto-second laser turning of fused silica with a diameter of $3.7 \mathrm{~mm}$ is investigated. The influence of the applied pulse energy is stated and the use of a trepanning optic during the turning process is characterized. Furthermore, comprehensive parameter studies in order to optimize roughness and to find the maximum achievable ablation rate are shown. Limited by the mechanical speed of the rotational axis, the highest feasible ablation rate is $19.5 \mathrm{~mm}^{3} / \mathrm{min}$. A roughness of $\mathrm{R}_{\mathrm{a}} 0.65 \mu \mathrm{m}$ are demonstrated.
\end{abstract}

DOI: $10.2961 / \mathrm{jlmn} .2021 .01 .2008$

Keywords: ultra-short laser pulses, laser turning, fused silica, ablation rate, process efficiency

\section{Introduction}

Fused silica is used in a broad variety of applications. Whether it is in optoelectronic devices, communication technology or in mere optics [1-3], fused silica is a crucial component for many industrial applications. The fabrication is based on melting quartz sand and casting the melt into the according form. After the solidification, a post-processing in terms of forming and re-shaping is an intricate procedure. The hard and brittle properties of the material make mechanical machining difficult.

Recently, the use of lasers, in particular ultra-short pulsed lasers, has come into focus for machining transparent materials [4]. By irradiating the material with ultra-short laser pulses of high energy density, material ablation takes place before the introduced energy can be passed on to the surrounding material, enabling a thermally neutral and contact free method of fabrication for freely selectable geometries $[5 ; 6]$.

Herman et al. [7] investigated ultrafast laser ablation of fused silica for its prospects in shaping photonic components. A processing window for shaping 3-dimensional surface structures was defined and surface roughnesses suitable for optical devices have been accomplished. Ahmed et al. [8] optimized the machining of grooves for superior breaking and edge quality in fused silica plates. Schwarz et al. [9] demonstrated a two-step process for manufacturing cylindrical lenses by combining ultra-short laser ablation of fused silica and subsequent polishing by $\mathrm{CO}_{2}$ laser to realize optical quality of the surface. Bliedtner et al. [10] described the generation of freeforms in different glass substrates by using an ultra-short pulsed laser. Boerner et al. [11] investigated angle-dependent ablation of dielectric rods, namely sapphire and fused silica. The studies showed that the penetration depth of ultra-short laser pulses in dielectrics do not alter with the angle of incidence. Furthermore it is stated that the ablation threshold fluence is inversely proportional to the cosine of the incidence angle. Bergner et al. [12] investigated high-aspect and high-quality in-volume modifications of glass. Using laser bursts and a Gaussian-Bessel beam via an axicon, smooth and even modification traces with a length of up to $7.4 \mathrm{~mm}$ can be inscribed. Recently, the fabrication of a phase-shifted fiber Bragg grating in a single mode fiber by inscribing two fiber Bragg gratings, of which one is slightly tilted, was demonstrated by Sun et al. [13]. When it comes to laser turning, the prevalent applications are conditioning, dressing and profiling of grinding wheels. Thereby, the surface of the grinding wheels are refaced by either melting off a surface layer or by precisely generating pre-specified flank geometries into the surface [14-19]. Ackerl et al. [20] also demonstrated how tangential machining with ultrashort laser pulses can be used to manufacture dental ceramic implants.

In this study, a shaping operation by tangential irradiation with ultra-short laser pulses of fused silica rods is presented. Thereby, the work piece rotates around its own axis while the laser beam is guided tangentially alongside the part, thus realizing the desired geometry. This approach enables the generation of rotationally symmetric parts without exerting mechanical forces onto the specimen, what makes it ideal for processing brittle materials. By machining the work 
piece tangentially, the profiling is, in general, achieved by positioning and feeding the laser beam. Therefore, the arrangement is self-limiting in terms of material removal.

\section{Materials and methods}

\subsection{Laser system}

The experiments were carried out on a five-axis laser micro machining station (GL.evo, GFH GmbH Germany) as illustrated in. Fig. 1. The $\mathrm{X}$ and $\mathrm{Z}$ axes realize the positioning of the laser beam while the $\mathrm{Y}$ and $\mathrm{C}$ axes perform the feeding and the rotating motion, respectively. The repeat accuracy of the $\mathrm{X}$ and $\mathrm{Y}$ stage is $1 \mu \mathrm{m}$, of the $\mathrm{Z}$-axis $3 \mu \mathrm{m}$ and those of the rotation axes $A$ and $C$ are 4 arcsec and 2 arcsec, respectively. The trepanning optic enables a circular motion of the laser spot at an adjustable diameter of up to $390 \mu \mathrm{m}$ at a speed of up to $28000 \mathrm{rpm}$.

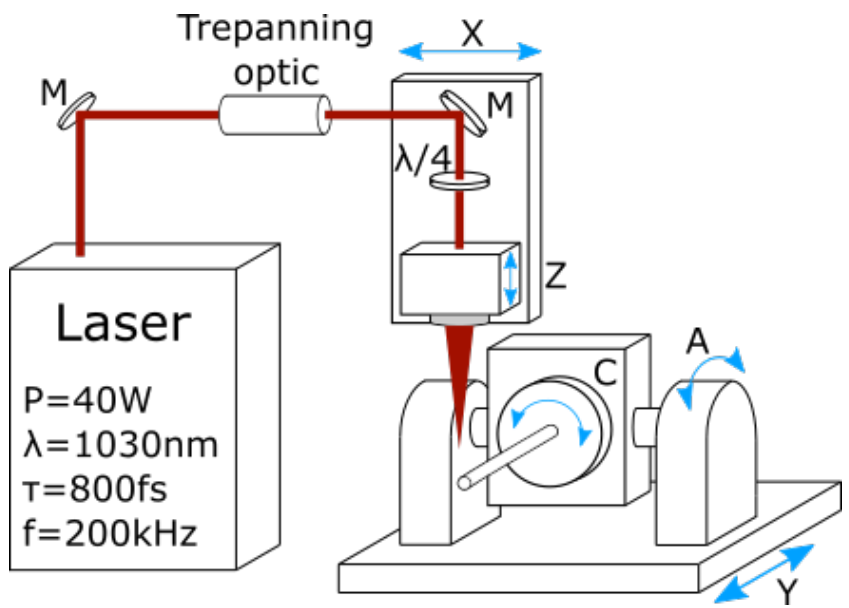

Fig. 1 Setup of laser micro machining station.

As laser source, an ultra-short pulsed laser system (TruMicro 5050 Femto Edition, Trumpf GmbH Germany) with a maximum average output power of $40 \mathrm{~W}$ at a wavelength of $1030 \mathrm{~nm}$ was used. With a pulse length of $800 \mathrm{fs}$ and a repetition rate of $200 \mathrm{kHz}$ it delivers a maximum pulse energy of $200 \mu \mathrm{J}$. The laser beam is focused by a lens with a focal length of $60 \mathrm{~mm}$ resulting in a calculated focus spot diameter of $18 \mu \mathrm{m}$. A nozzle orifice diameter of $1.5 \mathrm{~mm}$ was chosen and the initial nozzle to work piece distance was set to $1 \mathrm{~mm}$. For processing gas, a constant flow of Nitrogen at approximately $30 \mathrm{l} / \mathrm{min}$ was chosen. To ensure a homogeneous energy absorption, the beam is guided through a quarter wave plate in order to generate circular polarization.

\subsection{Materials and process characterization}

For the studies, fused silica rods with an initial diameter of $3.7 \mathrm{~mm}$ were machined. The rods are processed tangentially while rotating around their own axis at a speed of $500 \mathrm{rpm}$, while the focused laser beam is guided parallel to the rotation axis of the work piece. The distance between the outer circumference and the laser tool path is referred to as offset (cf. Fig. 2). Depending on the initial work piece diameter, the offset defines, together with the applied pulse energy, the trepanning diameter and the laser spot diameter, the maximum feasible diameter reduction of the work piece and thus concludes the final contour of the geometry. Hence, this process is self-limiting in terms of material removal.

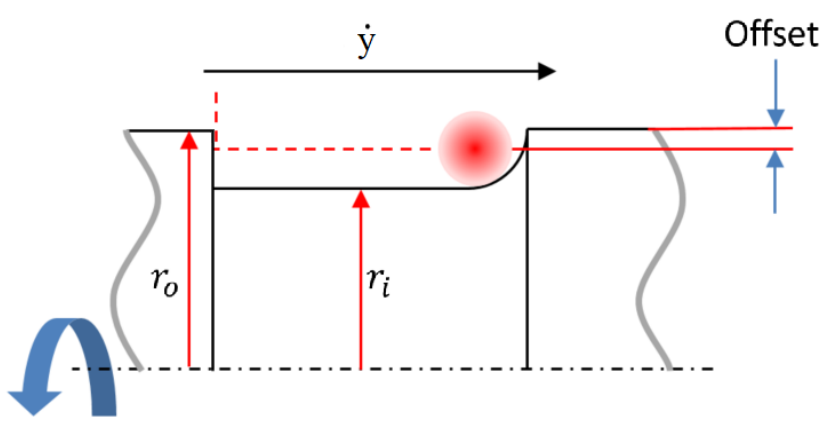

Fig. 2 Scheme of the geometry used in the parameter study (top view).

Throughout the process, the laser focal plane is kept on the same level as the rotation axis of the work piece.

The feasible feed rate for a continuous material removal in the tangential laser turning process strongly depends on the rotation speed $R$ of the workpiece and the laser spot diameter $d_{s}$. Exceeding the critical feed rate $\dot{y}_{c r i t}=R \cdot d_{s}$ results in a separation of the processing paths on the cylindrical surface of the work piece, potentially and inadvertently creating a thread.

The implementation of a trepanning diameter spreads the applied laser power over a wider area, thus artificially "enlarging" the laser spot diameter. This approach may therefore be used to potentially enhance the critical feed rate and improve the efficiency of the laser turning process.

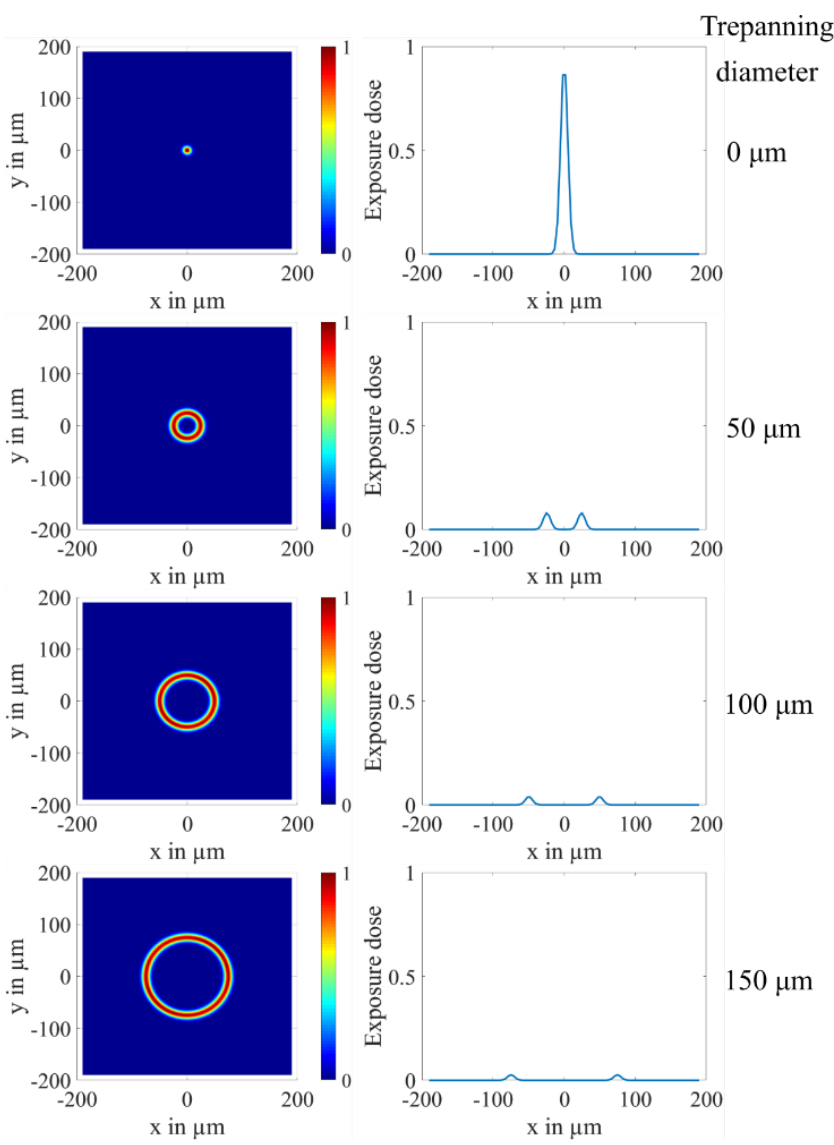

Fig. 3 Simulated long time exposure for one trepanning turn (left). Exposure cross-section through $\mathrm{y}=0$ (right). Scales standardized. 
As illustrated in Fig. 3, enlarging the trepanning diameter also results in lower intensities along the laser spot path due to lower pulse overlap. Hence, the laser feed rate has to be adjusted accordingly to ensure a homogenous ablation. Additionally, when applying a trepanning diameter greater than zero, an overlap gradient occurs along the exposure as depicted in Fig. 4.

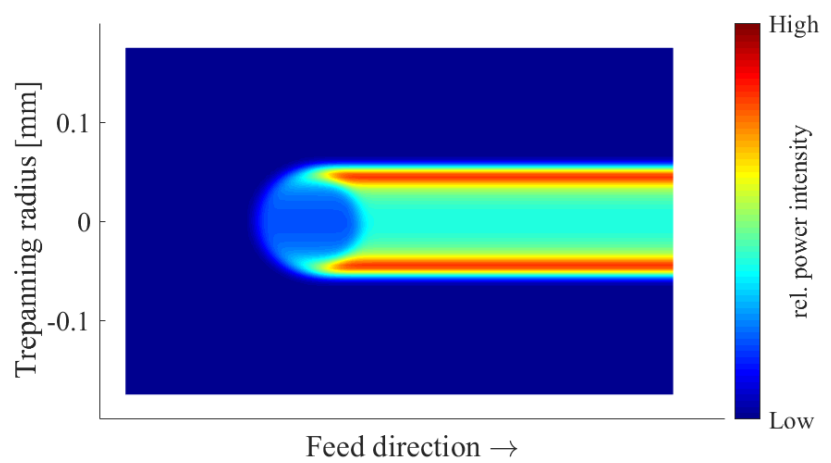

Fig. 4 Accumulated power intensity distribution for superimposed feed and trepanning motion. Exemplary image for trepanning diameter: $100 \mu \mathrm{m}$, trepanning speed: $28000 \mathrm{rpm}$, feed rate: $0.1 \mathrm{~mm} / \mathrm{s}$, frequency: $50 \mathrm{kHz}$

The superimposed motion of the laser feed and the trepanning optic causes an excessive amount of laser power to be deposited at the outer trepanning radius.

The illustrated intensity distribution in Fig. 3 and Fig. 4 is simplified for planar machining and should help to understand the relation between trepanning diameter, potentially deposited energy and feeding motion. In the laser turning process the machined parts are cylinder shaped, what makes the considerations of the intensity distribution more complex. All effects of defocusing and tilted surface are not included in this consideration.

For determining the ablation rate, the initial and final diameter are measured with an optical microscope. The resulting ablation rates $\dot{V}$ calculates as

$$
\dot{V}=\left(\left(r_{o}^{2}-r_{i}^{2}\right) \cdot \pi\right) \cdot \dot{y}
$$

with $r_{i}$ representing the inner (final) radius, $r_{o}$ the outer radius and $\dot{x}$ the relative speed of the laser alongside to the rotation axis (cf. Fig. 2).

The arithmetic average roughness $R_{a}$ was measured with a laser scanning microscope (Keyence VK - X200, Keyence Corp.) by scanning a total area of $280 \mu \mathrm{m}$ by $210 \mu \mathrm{m}$ on each sample and analyzing five individual squares with an area of $70 \mu \mathrm{m}$ by $70 \mu \mathrm{m}$ each. The result within each square consists of an average line roughness.

\section{Results and discussion}

\subsection{Ablation rates and efficiency}

In order to determine the achievable ablation rate for the laser turning process of fused silica, a study of various trepanning diameters at increasing pulse energies is conducted. The results are summarized in Fig. 5.

As expected, increasing pulse energy results in increasing ablation rates at all applied trepanning diameters. Obviously, a larger trepanning diameter contributes to an increased ablation rate within the investigated regime as well.

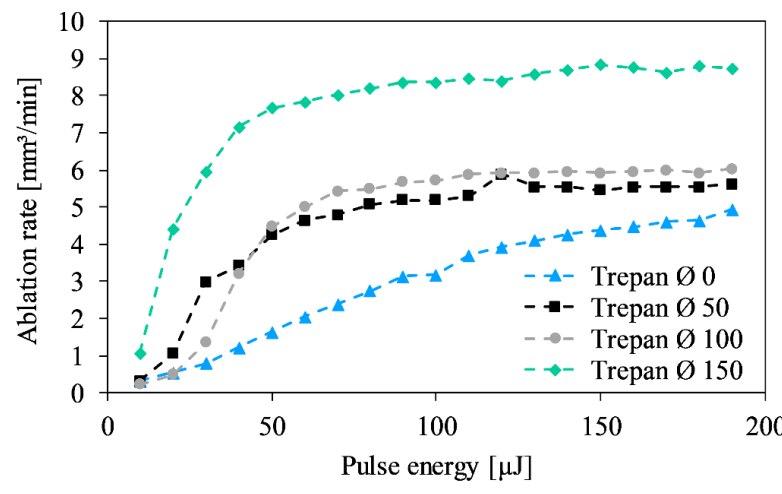

Fig. 5 Ablation rates versus pulse energy for varying trepanning diameters. Offset: $50 \mu \mathrm{m}$, feed rate: $0.01 \mathrm{~mm} / \mathrm{s}$.

The highest ablation rate value is reached at around $8.8 \mathrm{~mm}^{3} / \mathrm{min}$ with a trepanning diameter of $150 \mu \mathrm{m}$. Although a trepanning diameter of $150 \mu \mathrm{m}$ exceeds the offset of $50 \mu \mathrm{m}$, hence missing the material for roughly $27 \%$ of the trepanning revolution, a considerable rise of the ablation rate can be observed. We attribute this behavior to the decrease in pulse overlap, resulting in a decreased particle shielding effect as well as to the larger spot diameter on the material surface as a result of the increasing trepanning diameter and the tilted surface. Through this increase in spot size, more pulses of the optimal fluence irradiate the work piece, resulting in a higher ablation rate. The optimal Fluence for ablating fused silica is at around $2 \mathrm{~J} / \mathrm{cm}^{2}$ [21].

Self-limitation of the process is reached at pulse energies of $90 \mu \mathrm{J}, 110 \mu \mathrm{J}$ and $150 \mu \mathrm{J}$ for the trepanning diameters $50 \mu \mathrm{m}, 100 \mu \mathrm{m}$ and $150 \mu \mathrm{m}$, respectively.

The ablation efficiency of the process, defined by the ratio of ablation rate and applied laser power, is depicted in Fig. 6.

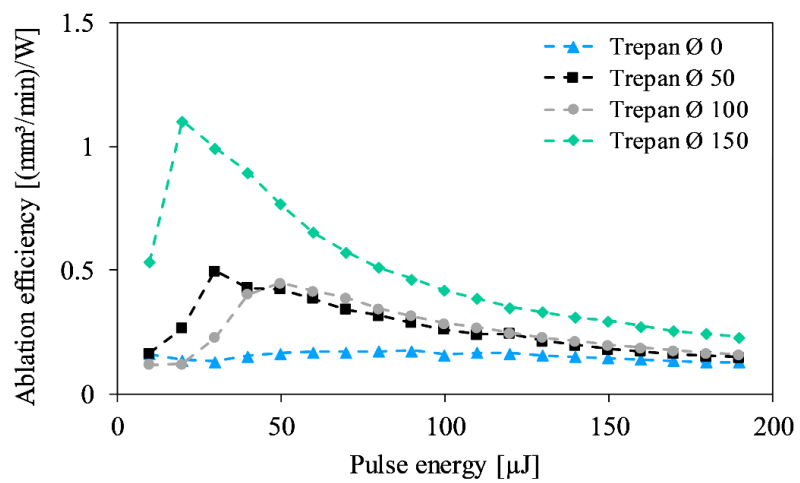

Fig. 6 Ablation efficiency versus pulse energy for varying trepanning diameters. Offset: $50 \mu \mathrm{m}$, feed rate: $0.01 \mathrm{~mm} / \mathrm{s}$.

The efficiency for an applied trepanning diameter of zero $\mu \mathrm{m}$ is relatively constant at around $0.15\left(\mathrm{~mm}^{3} / \mathrm{min}\right) / \mathrm{W}$ for increasing pulse energy. The efficiencies for trepanning diameters of $50 \mu \mathrm{m}$ to $150 \mu \mathrm{m}$, however, show a distinct peak between pulse energies of $20 \mu \mathrm{J}$ and $50 \mu \mathrm{J}$ before a steady decrease. This is assumed to be the point where the threshold fluence of the material, with respect to the angle of incidence, reaches its optimum. The maximum efficiency of about $1.1\left(\mathrm{~mm}^{3} / \mathrm{min}\right) / \mathrm{W}$ is reached at a pulse energy of $20 \mu \mathrm{J}$ and at a trepanning diameter of $150 \mu \mathrm{m}$. In compari- 
son to this value, typical values for normal incidence ablation processes are between 0.4 and $0.6\left(\mathrm{~mm}^{3} / \mathrm{W}\right) / \mathrm{min}[21$; 22].

To find the maximum feasible ablation rate, a pulse energy of $190 \mu \mathrm{J}$ and an offset of $200 \mu \mathrm{m}$ is applied for increasing feed rates in order to rule out the possibility of self-limitation of the process. The results are summarized in Fig. 7.

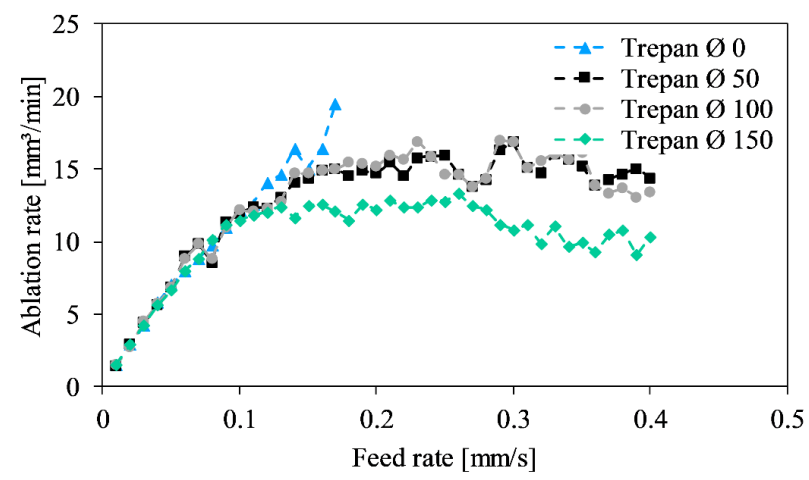

Fig. 7 Ablation rate versus feed rate for varying trepanning diameters. Offset: $200 \mu \mathrm{m}$, pulse energy: $190 \mu \mathrm{J}$

The highest value of around $19.5 \mathrm{~mm}^{3} / \mathrm{min}$ is reached with a trepanning diameter of zero and a feed rate of $0.17 \mathrm{~mm} / \mathrm{s}$, being limited by the critical feed rate for continuous material removal. Trepanning diameters of $50 \mu \mathrm{m}$ and $100 \mu \mathrm{m}$ reach an average value of around $15.1 \mathrm{~mm}^{3} / \mathrm{min}$ for feed rates of $0.2 \mathrm{~mm} / \mathrm{s}$ and above. A trepanning diameter of $150 \mu \mathrm{m}$ fluctuates at about $12.4 \mathrm{~mm}^{3} / \mathrm{min}$ for feed rates between 0.14 and $0.25 \mathrm{~mm} / \mathrm{s}$, before slowly decreasing for higher values. We assume that this decrease in ablation rate with increasing trepanning diameter combined with higher feed rates is due to the excessive spatial distribution of the applied laser power by this superimposed motion. Hence, not enough laser pulses per area are applied to trigger an efficient ablation procedure.

\subsection{Surface topography}

The accomplished roughness of the laser turning process of fused silica are summarized in Fig. 8. In this study, an increasing number of passes of the exposure path is implemented.

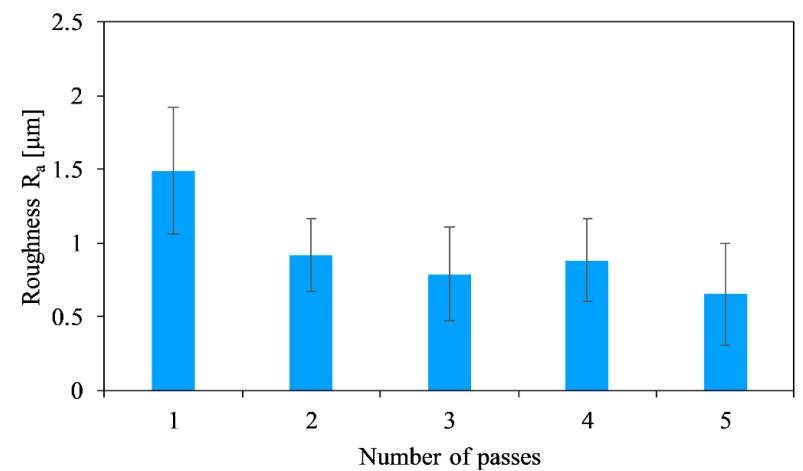

Fig. 8 Roughness at a trepanning diameter of $0 \mu \mathrm{m}$ and increasing number of passes. Offset: $0 \mu \mathrm{m}$, pulse energy: $190 \mu \mathrm{J}$, feed rate: $0.1 \mathrm{~mm} / \mathrm{s}$.

By applying a trepanning diameter of zero $\mu \mathrm{m}$, which is similar to using no trepanning optic at all, the most homog- enous pulse overlap throughout the exposure path can be accomplished. The data shows that, in general, it is beneficial to apply at least one additional pass to even out the ablated surface. The smoothest result with an arithmetic average roughness $R_{a}$ of $0.65 \mu \mathrm{m}$ is found after five consecutive passes. The error bars represent the root mean square deviation for five analyzed measurement areas on each sample surface.

\subsection{Geometry}

Within the boundaries of rotational symmetry, the shaping of material in laser turning is freely selectable. An example for feasible features on a laser turned work piece is given in Fig. 9.

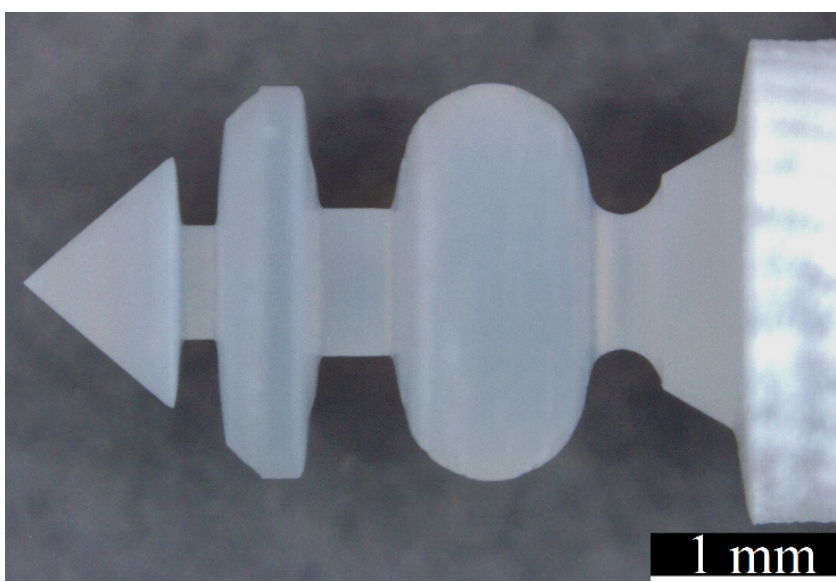

Fig. 9 Image of exemplary geometry

Spheres, arcs, clearances or angles are easily realizable by this process. Perpendicular angles, however, are either limited by the focal spot radius or applied trepanning diameter. Similar to mechanical turning, the laser turning process can be divided in two different processing steps: roughing and smoothing. While roughing removes most of the material, the subsequent smoothing of the work piece evens out the surface topography. In contrast to mechanical turning, both processing steps can be accomplished with the same tool, circumventing tool-setting time.

\section{Conclusion}

In this report the process of tangential laser turning of fused silica using ultra-short laser pulses was investigated. Intention of the studies was the investigation of the influence of pulse energy onto the feasible ablation rate as well as the impact of applied trepanning diameters onto the process efficiency. A maximum ablation rate of $19.5 \mathrm{~mm}^{3} / \mathrm{min}$ was achieved and a process efficiency of $1.1\left(\mathrm{~mm}^{3} / \mathrm{min}\right) / \mathrm{W}$ was demonstrated. For both cases a higher trepanning diameter has proven to be beneficial. A study of generated roughness's showed that in the laser turning process of fused silica, at least one additional smoothing step is commendable in order to achieve a lower roughness profile. Here, a minimum roughness $R_{a}$ of $0.65 \mu \mathrm{m}$ was achieved after five consecutive passes of the exposure path. Furthermore, the influence of the laser spot overlap gradient based on the superimposed trepanning and feeding motion was pointed out. 


\section{References}

[1] J. Zheng, C. Zhou, J. Feng, and B. Wang: Opt Lett, 14, (2008) 1554.

[2] S. Schwarz, S. Rung, C. Esen, and R. Hellmann: Opt. Express, 18, (2018) 23287.

[3] H.-K. Choi, J. Ryu, C. Kim, Y.-C. Noh, I.-B. Sohn, and J.-T. Kim: J. Laser Micro/Nanoengin., 3, (2016) 341.

[4] W. Watanabe, Y. Li, and K. Itoh: Opt. Laser Technol., 78, (2016) 52.

[5] M. Lenzner, J. Krüger, S. Sartania, Z. Cheng, C. Spielmann, G. Mourou, W. Kautek, and F. Krausz: Phys. Rev. Lett., 18, (1998) 4076.

[6] B. C. Stuart, P. S. Banks, M. D. Perry, M. D. Feit, R. S. Lee, F. Roeske, J. P. Armstrong, H. T. Nguyen, and J. A. Sefcik: Proc. SPIE, 3269, (1998) 57.

[7] P. R. Herman, A. Oettl, K. P. Chen, and R. S. Marjoribanks: Proc. SPIE, 3616, (1999) 148.

[8] F. Ahmed and M. S. Lee: Ultrahort Laser Applications of 7th CLEO-Pacific Rim, (2007) TuB2_3.

[9] S. Schwarz and R. Hellman: J. Laser Micro/Nanoengin., 12, (2017) 76.

[10] J. Bliedtner, C. Schindler, M. Seiler, S. Wächter, M. Friedrich, and J. Giesecke: Laser Tech. J., 5, (2016) 46.

[11] P. Boerner, M. Hajri, T. Wahl, J. Weixler, and K. Wegener: J. Appl. Phys., 23, (2019) 234902.

[12] K. Bergner, M. Müller, R. Klas, J. Limpert, S. Nolte, and A. Tünnerman: Appl. Opt., 21, (2018) 5941.

[13] X. Sun, L. Zeng, H. Du, X. Dong, Z. Chang, Y. Hu, and J. Duan: Opt. Laser Technol., 124, (2020) 105969.

[14] A. H. A. Lutey, A. Fortunato, F. Zanini, and S. Carmignato: Lasers Manuf. Mater. Process., 3, (2016) 158.

[15] M. Mukhopadhyay and P. K. Kundu: Int. J. Mechatron. Manuf. Syst., 11, (2018) 167.

[16] K. Wegener, H.-W. Hoffmeister, B. Karpuschewski, F. Kuster, W.-C. Hahmann, and M. Rabiey: CIRP Ann. Manuf. Technol., 60, (2011) 757.

[17] N. Ackerl, M. Warhanek, J. Gysel, and K. Wegener: Mater. Des., 189, (2019) 108530.

[18] G. Chen, S. Cai, and C. Zhou: Diam. Relat. Mater., 60, (2015) 99.

[19] C. Genyu, W. Yi, P. YanBo, and W. Yanyi: Int. J. Adv. Manuf. Technol., 104, (2019) 4077.

[20] N. Ackerl, M. Warhanek, J. Gysel, and K. Wegener: J. Eur. Ceram. Soc., 39, (2019) 1635.

[21] S. Schwarz, S. Rung, C. Esen, and R. Hellmann: Proc. SPIE, 11270, (2020) 112701E.

[22] N. Hodgson, S. Heming, A. Steinkopff, H. Haloui, and T. S. Lee: Lasers in Manufacturing Conference, (2019) 151.

(Received: June 26, 2020, Accepted: February 1, 2021) 